\title{
A Solenoidal Capture System For Neutrino Production *
}

\author{
M. Diwan, S. Kahn, R. B. Palmer, BNL, Upton, NY
}

\section{Abstract}

This paper describes the use of a high field solenoidal magnet to capture secondary pions from the production target. The captured pions subsequentially decay to produce the neutrino beam. A pion capture system using a high field solenoid magnet has been proposed for the muon collider[1]. This technology would also be available for neutrino beam production. It will be shown that a high field solenoid would produce a larger flux of neutrinos with energy, $E_{\nu}<1.3 \mathrm{GeV}$, than a neutrino beam produced with a horn system. The $\nu_{e}, \bar{\nu}_{e}$ flux contamination in the solenoid neutrino beam is only $0.15 \%$.

\section{INTRODUCTION}

The recent paper from the Super Kamiokande Collaboration[2] [3] indicating evidence for oscillations of atmospheric neutrinos has created interest in verifying the results with an accelerator based neutrino experiment. The probability of oscillation of one species of neutrino into another is proportional to $\sin ^{2}\left[\frac{1.27 \Delta m^{2} L}{E_{\nu}}\right]$. For oscillations in the small $\Delta m^{2}$ region it is advantageous to have either or both a long distance or small $E_{\nu}$. A high field solenoid can capture all pions produced from proton interactions on a target up to a transverse momentum, $P_{T}$, determined by the field, $B_{s}$, and the solenoidal radius, $R_{s}$. The pion capture solenoid (currently) proposed for the muon collider has $B_{s}=20$ tesla and $R_{s}=8 \mathrm{~cm}$. These parameters would limit the capture to pions with $P_{T}<0.225 \mathrm{GeV} / \mathrm{c}$ which would keep only $33 \%$ of the pions. Since muons from low $P_{T}$ pions can be more efficiently cooled, these parameters are reasonable for a muon collider. For neutrino production a larger solenoid aperture is preferred.

The solenoid field is 20 tesla in the vicinity of the target. The gradient of field, as it falls off moving away from the target, provides focussing by increasing the longitudinal momentum while decreasing the transverse momentum. The form of the field along the longitudinal axis is

$$
B_{s}(z)=\frac{B_{o}}{1 .+a z}
$$

where $B_{o}$ is the nominal field at the target and $a$ is a parameter that determines $\nabla \vec{B}$ and consequently the focussing. A solenoid tends to focus low momentum pions better than high momentum pions. The $\nu$ flux from solenoidally captured pions will dominate the $\nu$ flux from a horn beam at low neutrino energy. There is an additional contribution of $\bar{\nu}$ from a solenoid since the solenoid does not sign select as a horn does.

\footnotetext{
* Work performed under Contract No. DE-AC02-76CH00016 with the U.S. Department of Energy.
}

\section{SIMULATION}

A simulation study of solenoid focussed pion beam for $\nu$ production was made using GEANT[4]. The GEANT model used in the (now defunct) BNL long baseline experiment[5] was used as a basis. The two horn system in the GEANT model was replaced by the high field solenoid. The solenoid is 20 meters long with the radius varying as

$$
R(z)=R_{o} \sqrt{1+a z}
$$

where $R_{o}$ is the radius of the solenoid in the vicinity of the target. $R_{o}=16 \mathrm{~cm}$ is chosen so that all $\pi \mathrm{s}$ with $P_{T}<$ $0.45 \mathrm{GeV} / \mathrm{c}$ are kept. This aperture will capture $75 \%$ of the $\pi$ s produced. $a$ is chosen to give the proper focussing. Choosing $a=0.05 \mathrm{~cm}^{-1}$ will give a radius of $160 \mathrm{~cm}$ at the far end of the solenoid.

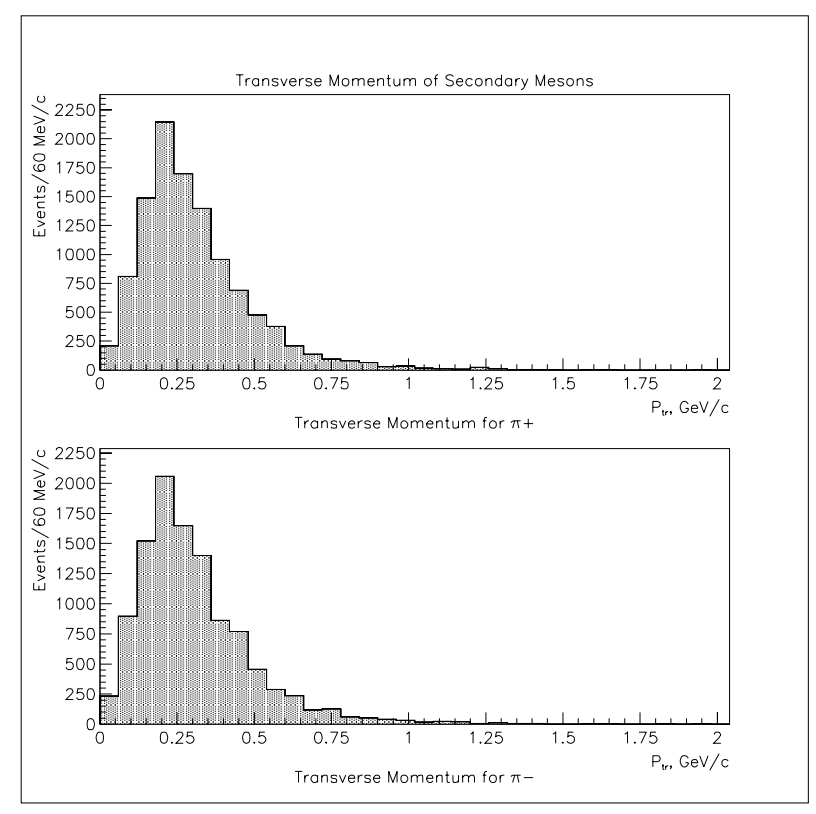

Figure 1: Transverse momentum distribution of secondary pions from the target using the FLUKA option in GEANT. $P_{T}$ is in $\mathrm{GeV} / \mathrm{c}$.

The solenoid for computational purposes is described by a sequence of 100 current rings with radius given by Eq 2 and spacing between the rings as approximately inversely proportional to the field strength. The current in the rings is varied so as to obtain the desired field on the axis. The magnetic field is obtained by summing the contribution from each ring where the field from each ring is calculated from an analytic expression[6]. This procedure for field computation is accurate and manifestly satisfies Maxwell's equation. It does not suffer from the descretation errors that would be present in using a field map for a 20 meter long 
expanding radius solenoid. It is however a very time consuming calculation.

In this study we use a $28.3 \mathrm{GeV}$ proton beam with a circular transverse profile with $\sigma_{r}=0.8 \mathrm{~mm}$ and $\sigma_{r^{\prime}}=0$. The beam is incident on a copper target with $1 \mathrm{~cm}$ radius and $22.5 \mathrm{~cm}$ length (1.5 interaction lengths). Table1 indicates the number of produced secondaries of each species per incident proton on target. The rates quoted are the same whether the secondaries are captured by a solenoid or a horn system. Table1 shows the secondary meson production rates for both the FLUKA[7] and GHEISHA[8] hadronic shower packages in GEANT. The rates using the FLUKA and GHEISHA are not consistent with each other. The FLUKA secondary $\pi$ s show less of a correlation between $P_{\perp}$ and $P_{\|}$. The FLUKA results are used for the analysis.

Table 1: Rates for secondary meson production at the target. Rates are for mesons produced per incident proton on target.

\begin{tabular}{||l||c|c||}
\hline Particle & Fluka & Gheisha \\
\hline$\pi^{+}$ & 2.83 & 3.73 \\
$\pi^{-}$ & 2.49 & 3.39 \\
$K^{+}$ & 0.13 & 0.09 \\
$K^{-}$ & 0.06 & 0.03 \\
$K_{L}$ & 0.10 & 0.07 \\
$K_{S}$ & 0.10 & 0.06 \\
\hline
\end{tabular}

After the secondary mesons are produced they are transported through the field which captures and focuses them. The produced $\pi \mathrm{s}$ can either decay in the field or in the decay tunnel following the solenoid or interact it the walls of the solenoid or tunnel. Fig 2 shows the momentum spectrum of the decaying and interacting $\pi \mathrm{s}$. The decay length for lower momentum $\pi \mathrm{s}$ is shorter, we expect the decaying $\pi$ s to have lower momentum. Fig 2 also shows the decay (interaction) position of the $\pi \mathrm{s}$. This figure shows that the $\pi \mathrm{s}$ that interact will preferentially do that in the solenoid (axial position less than 20 meters) by hitting the wall of the solenoid. For computational efficiency, each decaying meson is allowed to decay 10 times producing neutrinos in different parts of phase space. The neutrinos produced are appropriately weighted to preserve the normalization.

\section{SPECTRUM}

Fig 3 shows the neutrino spectrum at 1 kilometer from the target along the incident beam direction. The spectrum is given in units of $\nu \mathrm{s} /\left(\mathrm{GeV} \cdot \mathrm{m}^{2} \cdot \mathrm{POT}\right)$, where POT refers to protons on target. Fig 3 shows three spectral curves. The dashed curve corresponds to the solenoidal focussed beam described above. It shows the $\nu$ flux is largest below $1.2 \mathrm{GeV}$ falling off sharply above that. The dotted curve shows the $\nu$ spectrum of the two horn focus beam that was designed to be used for the BNL Long Baseline experiment. This beam peaks with $E_{\nu} \approx 1.5 \mathrm{GeV}$ and

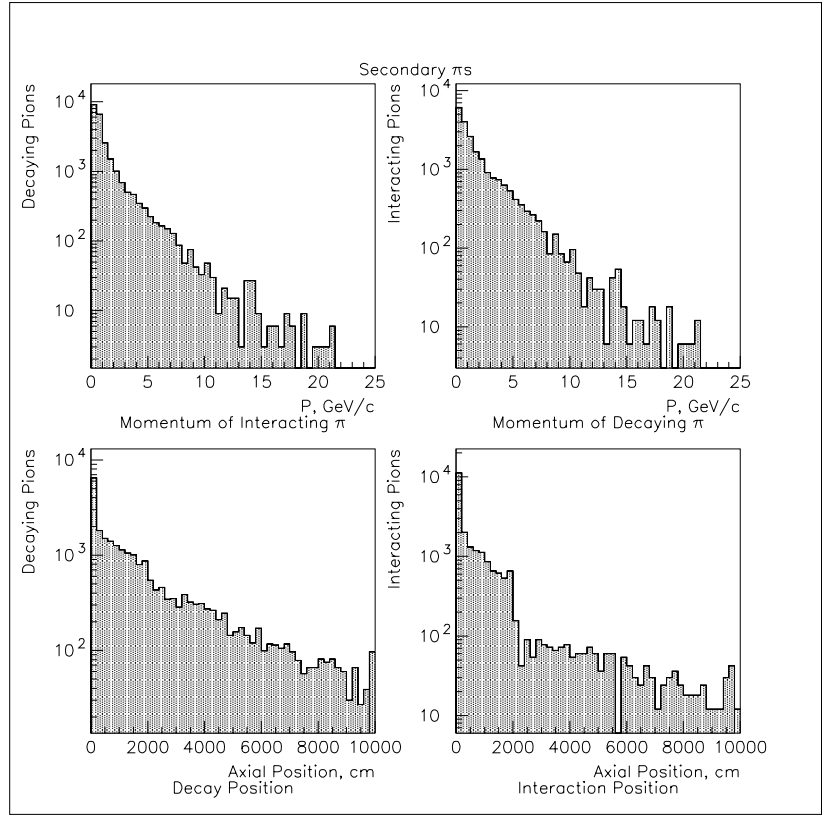

Figure 2: Momentum and decay(interaction) point of decaying (interacting) pions.

extending to about $4 \mathrm{GeV}$. The solid curve represents the spectrum of the so-called perfect focussed neutrino beam. For the perfect focussed beam it is assumed that each secondary meson exiting the target has its direction parallel to the incident proton beam. The perfect beam would have the largest $\nu$ spectrum imaginable, but it is not physically achievable. The $\nu_{\mu}$ flux from the solenoidal focussed beam

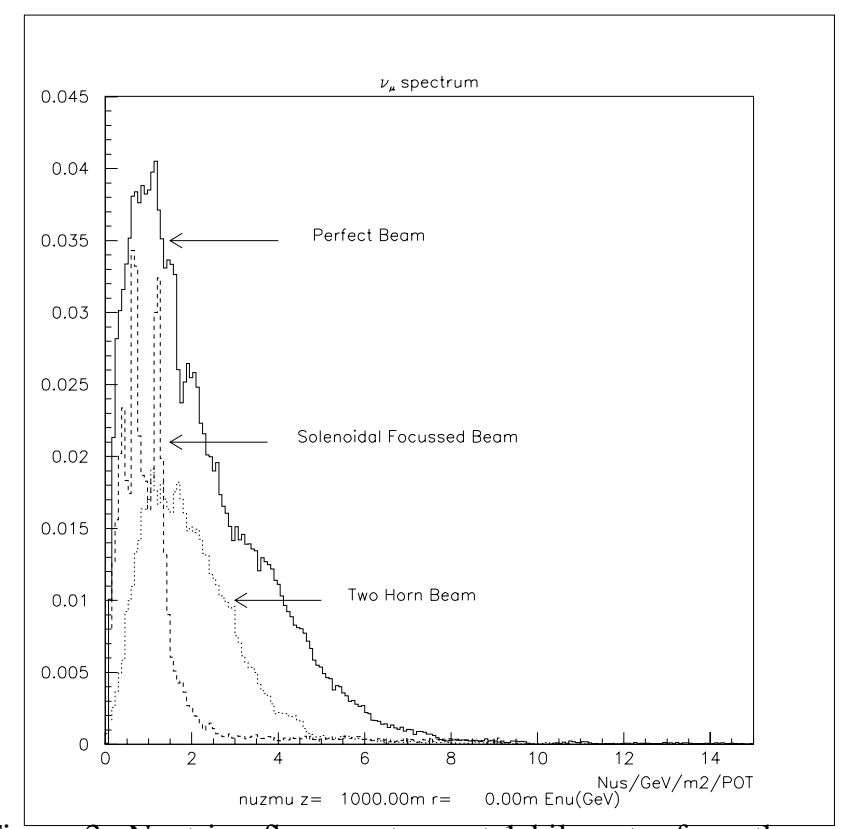

Figure 3: Neutrino flux spectrum at 1 kilometer from the target along the beamline. The three curves indicate the solenoidal focussed beam (dashed), two horn beam (dotted) and perfect focussed beam (solid).

dominates the flux from the two horn beam below $1.3 \mathrm{GeV}$. 
Table 2 compares the integrated $\nu_{\mu}$ flux per proton on target at a detector situated 1 kilometer from the target. $\nu_{\mu} \mathrm{s}$ with $E_{\nu}<0.45 \mathrm{GeV}$ are not interesting since the cross section to produce a charge current event is very small and consequentially the normalization for neutrino oscillations would be difficult. Table 2 separates the integrated flux into energy ranges $0.45<E_{\nu}<1.28 \mathrm{GeV}$ where the solenoidal focussed beam is larger than the horn beam and the region $E_{\nu}>1.28 \mathrm{GeV}$ where the horn beam beam is effective. In the region $0.45<E_{\nu}<1.28 \mathrm{GeV}$, the solenoidal $\nu$ beam will produce $67 \%$ more $\nu_{\mu}$ than the two horn system.

Table 2: Integrated $\nu_{\mu}$ flux at a detector 1 kilometer from the target. The units are number of $10^{-3} \nu$ s per $m^{2}$ per proton on target.

\begin{tabular}{||l||c|c|c||}
\hline Range, GeV & Solenoid & 2 Horn & Perfect \\
\hline All & 0.453 & 0.597 & 1.454 \\
$0.45<E_{\nu}<1.28$ & 0.250 & 0.150 & 0.409 \\
$E_{\nu}>1.28$ & 0.146 & 0.433 & 0.954 \\
\hline
\end{tabular}

A solenoid will focus both $\pi^{+}$and $\pi^{-}$whereas a horn focussed beam will select the sign of the $\pi \mathrm{s}$. A neutrino experiment could use both the $\nu_{\mu}$ and $\bar{\nu}_{\mu}$ events. Fig 4.a shows

$$
1 \times[\nu \text { spectrum }]+\frac{1}{3} \times[\bar{\nu} \text { spectrum }]
$$

The $\frac{1}{3}$ corrects for the fact that the $\bar{\nu}$ cross section is (approximately in this energy range) one third of the $\nu$ cross section. Table 3 shows the integrated flux for the total neutrino flux.

Table 3: Integrated $\nu_{\mu}+\frac{1}{3} \bar{\nu}_{\mu}$ flux at a detector 1 kilometer from the target. The units are number of $10^{-3} \nu \mathrm{s}$ per $m^{2}$ per proton on target.

\begin{tabular}{||l||c|c|c||}
\hline Range, GeV & Solenoid & 2 Horn & Perfect \\
\hline All & 0.571 & 0.605 & 1.824 \\
$0.45<E_{\nu}<1.28$ & 0.317 & 0.152 & 0.523 \\
$E_{\nu}>1.28$ & 0.179 & 0.439 & 1.184 \\
\hline
\end{tabular}

This effective total flux for the solenoidal focussed beam is twice that of the two horn focussed beam in the energy range from 0.45 to $1.28 \mathrm{GeV}$. The $\nu_{e}$ and $\bar{\nu}_{e}$ contamination for the solenoidal focussed beam is expected to be small since $\mathrm{K}$ production is small at low $E_{\nu}$. The ratio of $\nu_{e}, \bar{\nu}_{e}$ flux to $\nu_{\mu}, \bar{\nu}_{\mu}$ flux is $0.15 \%$ for the solenoid beam and $0.78 \%$ for the two horn beam. Fig 4.b shows the $\nu_{e}$ spectrum of the solenoid and horn beams.

\section{CONCLUSIONS}

A solenoidal focussed neutrino beam would produce a larger $\nu$ flux in the interesting energy range where one would like to look for $\nu_{\mu} \rightarrow \nu_{e}$ oscillations. In the energy range $0.45<E_{\nu}<1.28$ the solenoid would produce $60 \%$ of the neutrino flux of the perfect beam. The solenoidal focussed beam would produce almost twice the number of

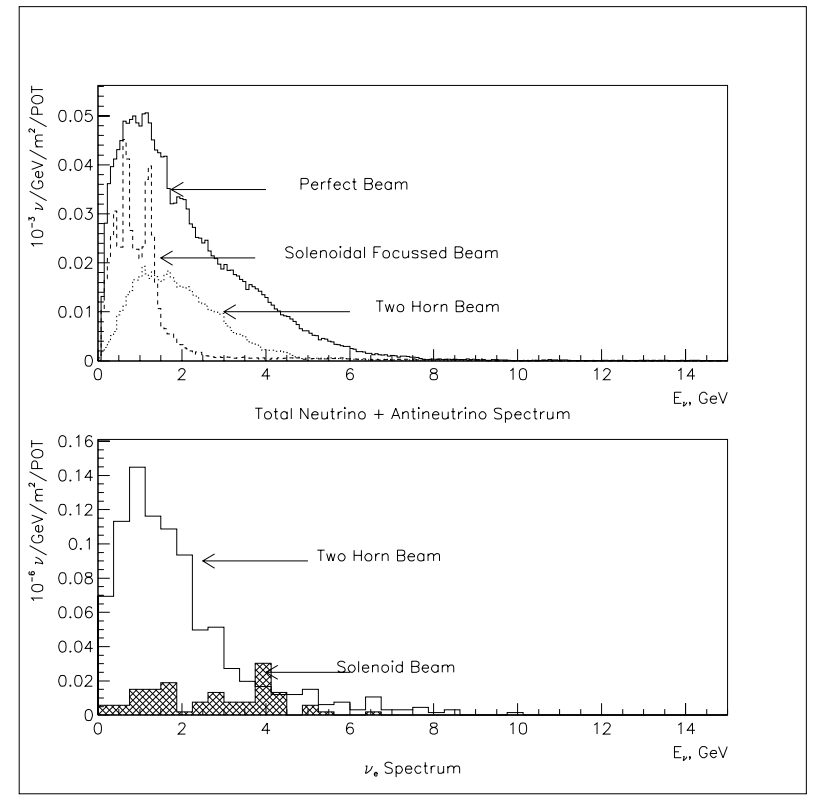

Figure 4: Part a: Sum of the effective spectra from $\nu$ and $\bar{\nu}$ at 1 kilometer from the target along the beamline. The figure sums the neutrino spectrum with weight 1 and the antineutrino spectrum with weight $\frac{1}{3}$. The three curves indicate the solenoidal focussed beam (dashed), two horn beam (dotted) and perfect focussed beam (solid). Part b: $\nu_{e}$ flux for the solenoidal focussed beam and horn focussed beam

$\nu+\bar{\nu}$ events as a horn focussed beam. The solenoidal focussed beam would have a much smaller $\nu_{e}$ contamination than the horn focussed beam. However, a 20 meter solenoidal capture and focussing magnet with a 20 tesla peak field would not be as cost effective as a standard two horn focus system. If however such a magnet were to be built for another application, such as a muon collider, it could also be used to produce high intensity low energy neutrino beam that would be ideal for $\nu_{\mu} \rightarrow \nu_{e}$ oscillations.

\section{REFERENCES}

[1] Muon Collider Status Report, to be submitted to Phys Rev. BNL-65623, FNAL-PUB-98/179, LBNL-41935

[2] Y. Fukuda, et al., Evidence of Oscillations of Atmospheric Neutrinos, Phys. Rev. Lett. 81, 1562 (1998)

[3] S. Hatakeyama et al., Measurement of the Flux and ZenithAngle Distribution of Upward Through-Going Muons in Kamiokande II + III, Phys. Rev. Lett. 81, 2016 (1998)

[4] GEANT Detector Description and Simulation Tool, CERN Program Writeup W5013

[5] Long Baseline Neutrino Oscillation Experiment, E889 Physics Design Report, BNL No. 52459, (1995)

[6] W.R. Smythe, Static and Dynamic Electricity, 2nd Edition (1950), p271

[7] P.A.Aarnio et al., FLUKA User Manual, Technical Report TIS-RP-190, CERN, (1987,1990)

[8] H.C.Fesefeldt, Simulation of Hadronic Showers, Physics and Applications, Technical Report PITHA 85-02, (1985) 